\title{
3D modelling of heating of thermionic cathodes by high-pressure arc plasmas
}

\author{
M S Benilov ${ }^{1}$, M Carpaij ${ }^{2}$ and M D Cunha ${ }^{1}$ \\ ${ }^{1}$ Departamento de Física, Universidade da Madeira, Largo do Município, 9000 Funchal, \\ Portugal \\ ${ }^{2}$ Lighting Technology Institute, University of Karlsruhe, Kaiserstr. 12, 76128 Karlsruhe, \\ Germany
}

Received 4 November 2005, in final form 7 March 2006

Published 5 May 2006

Online at stacks.iop.org/JPhysD/39/2124

\begin{abstract}
Numerical investigation of steady-state interaction of a high-pressure argon plasma with a cylindrical tungsten cathode is reported. A whole 'zoo' of very diverse modes of current transfer is revealed. Detailed results are given for the first five (three-dimensional) 3D spot modes, four of them branching off from the diffuse mode and one from the first axially symmetric spot mode. Divergences in the general pattern of solutions, which have been present in preceding works, are resolved. Hypotheses on stability of steady-state solutions, available in the literature, are analysed. It is found that these hypotheses provide an explanation of the fact that the transition between diffuse and spot modes is difficult to reproduce in the experiment but they do not explain the indication that it is the low-voltage branch of the first 3D spot mode that seems to occur in the experiment. Thus, the question of stability of steady-state solutions remains open: an accurate stability analysis, as well as additional experimental information is required.
\end{abstract}

(Some figures in this article are in colour only in the electronic version)

\section{Introduction}

Current transfer from high-pressure arc plasmas to thermionic cathodes may occur in a diffuse mode, when the current is distributed over the front surface of the cathode in a more or less uniform way, or in a spot mode, when most of the current is localized in one or more small areas (cathode spots). In a certain arc current range, either of the modes can occur. In the case of an axially symmetric cathode, the diffuse mode is axially symmetric while spot modes can be both axially symmetric (a spot at the centre of the front surface of the cathode) or three-dimensional (3D) (an off-centre spot or a system of two or more spots).

It was suggested some years ago [1] that an adequate theoretical description of multiple modes of current transfer to thermionic cathodes does not necessarily involve essentially different physical mechanisms but is rather a mathematical question of finding non-unique solutions: an adequate theoretical model of current transfer to hot cathodes must in some cases allow different steady-state solutions to exist for the same conditions, which describe different modes of current transfer. It was shown that such multiple solutions exist in the framework of the model of nonlinear surface heating and a general pattern of solutions describing various modes was suggested on the basis of bifurcation analysis and general considerations.

In the subsequent years, the model of nonlinear surface heating was validated by an extensive comparison with the experiment [2,3] and has become a widely accepted tool for modelling of interaction of high-pressure arc plasmas with thermionic cathodes. At present, steady-state axially symmetric modes on axially symmetric cathodes have been (numerically) studied in detail and are understood relatively well (e.g. [2, 4, 5]); this applies to both diffuse and axially symmetric spot modes. Numerical results of 3D spot modes have started to appear only recently [6-8]. In [6], bifurcation points have been calculated in which $3 \mathrm{D}$ steady-state spot modes on axially symmetric cathodes branch off from axially symmetric modes. It was shown that 3D spot modes can branch off from both the diffuse mode and axially symmetric spot modes. The 3D transient spots on a cylindrical cathode were simulated in [7]. In [8], results of simulations were given for a $3 \mathrm{D}$ steady-state spot attached to a rounded edge of a cylindrical cathode. 
The physics of 3D spots should be similar to the physics of axially symmetric spots, which has been studied in detail in [5]. The importance of computational aspects of 3D simulations has considerably decreased in recent years due to the appearance of powerful computers and advanced software. There is, however, an aspect in the modelling of 3D spot modes which is still of primary interest, namely, finding the general pattern of different modes. Among other things, this pattern is critical for analysis of stability of various modes. It is also needed in order to permit the identification of a particular mode of current transfer observed in simulations or in experiment. Note that the available works diverge as far as this pattern is concerned: 3D spot modes reported in [8] break off at a certain current instead of turning back or joining other modes, which does not fit in the pattern $[1,6]$ and is not a behaviour typical of multiple solutions.

Establishing the pattern of different 3D modes of steadystate current transfer to thermionic cathodes represents the goal of this work. The outline of the paper is as follows. The model of nonlinear surface heating is briefly described in section 2. In section 3 , multiple solutions describing various modes are given and general features of $3 \mathrm{D}$ solutions are analysed. The diffuse mode and the first 3D spot mode under typical experimental conditions are calculated and analysed in section 4. Information on stability of steady states belonging to different modes, available in the literature, is analysed in section 5. Concluding remarks are given in section 6 .

\section{The model and its numerical realization}

A summary of equations for the theory of interaction of thermionic cathodes with high-pressure arc plasmas, based on the model of nonlinear surface heating, is given in a recent paper [9]. (Some additional theoretical materials and an online tool for simulation of the diffuse mode of current transfer developed in the framework of this theory can be found on the Internet [10].) The model can be briefly described as follows. A steady-state temperature distribution in the body of a cathode is considered. Joule heat production in the body of the cathode is neglected. The base of the cathode is maintained at a fixed temperature $T_{\mathrm{c}}$ by external cooling and the rest of the cathode surface is in contact with the plasma or the cold gas and is heated or cooled respectively.

Mathematically, the problem amounts to solving the thermal-conduction equation

$$
\nabla \cdot(\kappa \nabla T)=0
$$

with the boundary condition

$$
T=T_{\mathrm{c}}
$$

at the base and with the nonlinear boundary condition

$$
\kappa \frac{\partial T}{\partial n}=q\left(T_{w}, U\right)
$$

at the rest of the cathode surface. Here $\kappa$ is the thermal conductivity of the cathode material, $n$ is a direction locally orthogonal to the cathode surface and directed outside the cathode and $q=q\left(T_{w}, U\right)$ is a given function of the cathode surface temperature $T_{w}$ and of the near-cathode voltage drop
$U$, which describes the density of the energy flux to the part of the cathode surface that is in contact with the arc plasma and the cold gas.

Functions $q=q\left(T_{w}, U\right)$ and $j=j\left(T_{w}, U\right)$ describing the density of electric current to the cathode surface are calculated by means of equations summarized in [9] (see also [2, 6, 11]) and are not discussed here. We only answer a frequently asked question of derivation of expressions given in the paper [11] for the densities of fluxes of the kinetic energy delivered to, or removed from, the cathode surface by different plasma species. These expressions are obtained by means of averaging the product of the particle kinetic energy times the particle velocity; a detailed derivation has been posted on the Internet [10]. Note that, since the average value of a product is not equal to the product of average values of multipliers, the density of flux of kinetic energy of particles whose distribution is assumed to be Maxwellian (which is the case of plasma electrons, the emitted electrons and neutral atoms leaving the cathode surface) is equal to $2 k T J$ rather than $3 k T / 2 J$ as one could expect intuitively (here $T$ is the temperature of the species in question, $J$ is the number density of the flux of this species and $k$ is the Boltzmann constant).

Equation (1) is written without accounting for Joule heat production in the cathode body. This approximation is justified provided that the electric power dissipated inside the cathode is much smaller than the power supplied to the cathode surface or, equivalently, that the voltage drop inside the cathode is much smaller than $U$. If the cathode operates in the diffuse mode then the voltage drop inside the cathode may be estimated by order of magnitude as $I \rho h / A$, where $I$ is the arc current, $\rho$ is the electrical resistivity of the cathode material, $h$ is the cathode height and $A$ is the area of the cathode cross section. Let us assume for the estimate that the arc current is $10 \mathrm{~A}$, the cathode height is $1 \mathrm{~cm}$ and the cathode cross section is a circle of radius $1 \mathrm{~mm}$. Electrical resistivity of tungsten increases from $5.3 \times 10^{-8} \Omega \mathrm{m}$ at room temperature to $94 \times 10^{-8} \Omega \mathrm{m}$ at $3000 \mathrm{~K}$ [12]; let us assume $\rho=10^{-6} \Omega \mathrm{m}$ for the estimate. Then $I \rho h / A=32 \mathrm{mV}$ and the Joule heating in the cathode body may be safely neglected. If the cathode operates in the spot mode then there are two components of the voltage drop inside the cathode: the voltage drop in the expansion zone (a domain inside the cathode adjacent to the spot in which the current density decreases from values typical for the spot to those of the order of $I / A$ ) and the voltage drop in the bulk of the cathode body, which is of the order of $I \rho h / A$. In order to get a reasonable estimate of the first component, let us assume that the current expansion is semi-spherical, then the corresponding voltage drop equals $I \rho / 2 \pi r_{*}$, where $r_{*}$ is the radius of the spot. Assuming $r_{*}=10 \mu \mathrm{m}$, one finds $I \rho / 2 \pi r_{*}=160 \mathrm{mV}$ and the Joule heating in the cathode body may be neglected in the spot mode as well.

The code developed for numerical solution of the abovedescribed problem consists of two modules. The first module calculates functions $q=q\left(T_{w}, U\right)$ and $j=j\left(T_{w}, U\right)$ by solving equations describing the near-cathode layer in a highpressure arc plasma which are summarized in [9]. Note that this module provides data in a wide range of values of surface temperature (up to $5000 \mathrm{~K}$ or higher) and near-cathode voltage drop (up to several hundred volts or higher) and for various plasma-producing gases (most of the pure monoatomic gases 
and a number of mixtures; see manual of the modelling tool [10]). This module is written in Fortran. The second module calculates the temperature distribution inside the cathode body and at the surface by solving the nonlinear boundary-value problem (1)-(3) in the cathode. This module is realized using the commercial finite element software FEMLAB.

The two ways of connecting the two modules are used in different versions of the code. One way consists of running the modules sequentially. In the framework of this approach, the first module generates files with data on functions $q=$ $q\left(T_{w}, U\right)$ and $j=j\left(T_{w}, U\right)$ and the second module makes use of these files. The other way consists of invoking the first module from inside the second one. In the framework of this approach, the first module is realized either as an executable file or, preferably, as a Matlab-compatible dll file.

\section{General features of 3D solutions}

Numerical results given in this work refer to a tungsten cathode in the form of a right circular cylinder (a rod) operating in an argon plasma. Data on thermal conductivity and emissivity of tungsten have been taken from [13] and [14], respectively; the value of $4.55 \mathrm{eV}$ was assumed for the work function of tungsten.

While dealing with temperature distributions possessing planar symmetry, one can restrict the calculation domain to half of the rod. In addition to savings in RAM and CPU time, this fixes the azimuthal position of the spot system and hence improves the convergence.

A finite element mesh automatically generated by FEMLAB was locally refined in the vicinity of each spot in order to obtain good accuracy. The number of finite elements routinely used in computations was around 40000 , which was presumably sufficient to obtain a solution to an accuracy of few per cent at worse.

In this section, results are given and discussed for a (tungsten) cathode of radius $R=2 \mathrm{~mm}$ and height $h=10 \mathrm{~mm}$; a geometry convenient for the illustration of general features of multiple solutions to the problem considered. The (argon) plasma pressure is assumed to be atmospheric.

\subsection{Current-voltage characteristics}

Current-voltage characteristics of various modes of current transfer to a tungsten cathode of this geometry operating in atmospheric-pressure argon plasma are shown in figure 1. A magnification of the central part of figure 1 is shown in figure 2 .

Current-voltage characteristics of the diffuse mode (which is axially symmetric) and the first axially symmetric spot mode (which corresponds to a spot at the centre of the front surface of the cathode) shown in figure 1 coincide with those calculated in [5] and are not discussed here. We only note that the current-voltage characteristic of the diffuse mode has two branches, one falling and the other rising, separated by a point of minimum, while the current-voltage characteristic of the first axially symmetric spot mode has two branches, a low-voltage one and a high-voltage one, separated by a turning point. Note that the diffuse mode and the first axially symmetric spot mode have been calculated in this work by means of the finite-element software FEMLAB and in [5] by

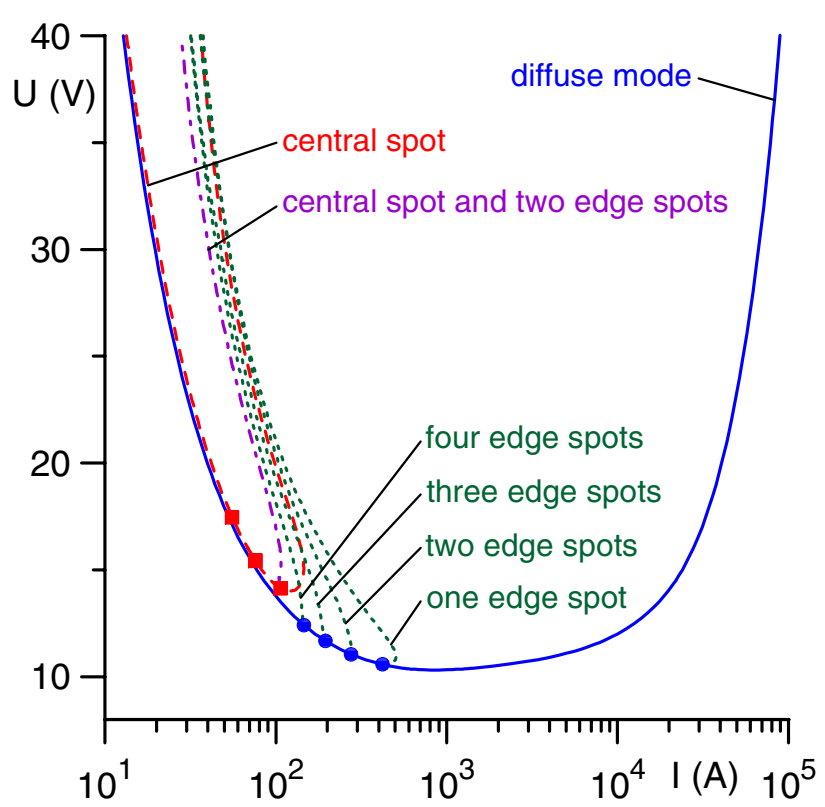

Figure 1. Current-voltage characteristics of different modes of current transfer. $R=2 \mathrm{~mm}, h=10 \mathrm{~mm}$. Solid line: diffuse mode. Dashed line: first axially symmetric spot mode. Dotted line: 3D spot modes which branch off from the diffuse mode. Dashed-dotted line: 3D spot mode which branches off from the first axially symmetric spot mode. Circles and squares: bifurcation points.

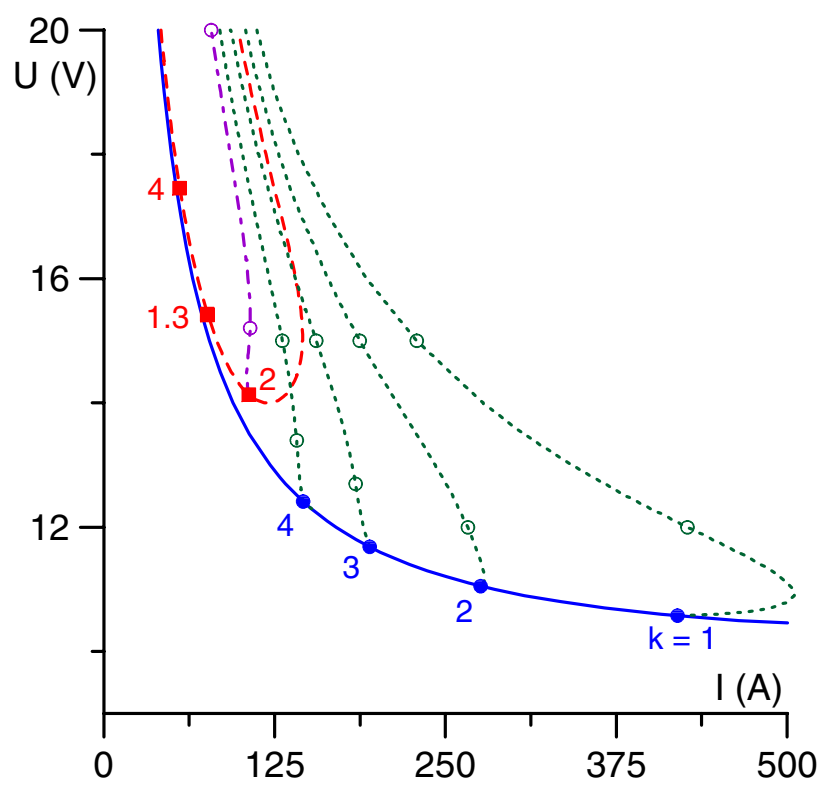

Figure 2. Transition between axially symmetric modes and 3D spot modes. $R=2 \mathrm{~mm}, h=10 \mathrm{~mm}$. Full circles: bifurcation points at which 3D modes branch off from the diffuse mode. Squares: bifurcation points at which 3D modes branch off from the first axially symmetric spot mode. $k$ determines the number of spots at the edge of the front surface of the cathode existing in each mode. Open circles: states of 3D spot modes which are shown in the subsequent figures.

means of a Fortran code implementing an iterative approach based on a finite-difference numerical scheme. Results given by the two codes coincide to a very high accuracy, which attests to accurate operation of both codes.

Also shown in figures 1 and 2 are bifurcation points positioned on the diffuse and first axially symmetric spot 
modes, calculated as described in [6]. We remind that, according to the general pattern established in $[1,6], 3 \mathrm{D}$ spot modes on an axially symmetric cathode branch off from axially symmetric modes. In other words, a value of electric current exists for every 3D spot mode such that at this current the cathode temperature distribution corresponding to this $3 \mathrm{D}$ mode turns axially symmetric and exactly coincides with the cathode temperature distribution corresponding to an axially symmetric mode. Such violations of unity of solutions are well known in mathematics and are termed bifurcations; in these terms, the above-mentioned value of electric current (as well as the corresponding value of $U$ ) may be called a bifurcation point. The azimuthal dependence of temperature distributions corresponding to $3 \mathrm{D}$ spot modes is described in the vicinity of bifurcation points by the factor $\cos k \phi$, where $k=1,2,3, \ldots$ and $\phi$ is the azimuthal angle. Values of $k$ corresponding to each bifurcation point are indicated in figure 2 (the bifurcation points corresponding to $k=1$ and $k=3$ positioned on the first axially symmetric spot mode coincide with the graphical accuracy). Also note that, strictly speaking, what branches off at each bifurcation point is a family of 3D spot modes rather than a single mode; however, these modes are identical to the accuracy of rotation and can therefore be considered as a single mode with an arbitrary azimuthal position of the spot system.

In contrast to the axially symmetric modes and bifurcation points, 3D spot modes shown in figures 1 and 2 have not been calculated previously. Each one of these modes branches off from an axially symmetric mode, in accord with the general pattern suggested in $[1,6]$. Five 3D spot modes are represented in figures 1 and 2, four of them branching off from the diffuse mode and one from the first axially symmetric spot mode. Points at which this branching occurs coincide exactly with bifurcation points that are predicted by bifurcation theory [6] and are shown in figures 1 and 2 by full circles and squares.

At large distances from the bifurcation points, all spot modes tend towards the region of small currents and high voltages, i.e. approach the axis of voltages. General considerations explaining this behaviour have been given in [1]. On the other hand, behaviour of different modes in the vicinity of the bifurcation points is essentially different: while the first two 3D modes bifurcate from the diffuse mode with a positive derivative $\mathrm{d} U / \mathrm{d} I$, the third and fourth $3 \mathrm{D}$ modes bifurcate from the diffuse mode with a negative derivative; the first 3D mode bifurcates from the first axially symmetric spot mode with a negative derivative.

In the vicinity of bifurcation points, near-cathode voltages and thermal regimes of 3D modes are close to those of axially symmetric modes. On the other hand, the fact that near-cathode voltages of two modes are close does not necessarily mean that thermal regimes of these modes are also close. For example, the current-voltage characteristic of the low-voltage branch of the first axially symmetric spot mode in figure 1 is very close to the characteristic of the diffuse mode; however, thermal regimes of these two modes are different.

\subsection{Thermal regime of the cathode}

Evolution of the temperature distributions along the different modes represented in figures 1 and 2 is shown in figures 3-9. Evolution of axially symmetric distributions, shown in figures 3 and 4, has been analysed in [5] and is not discussed here (these figures are shown here for the sake of completeness). Evolution of 3D temperature distributions is shown in figures 5-9. The first state shown for each mode corresponds to the bifurcation point at which this mode branches off from the diffuse mode or from the first axially symmetric spot mode, the second and third states are indicated in figure 2 by open circles and the fourth state for each mode corresponds to the maximal voltage drop value shown in figure 1 , i.e. to $U=40 \mathrm{~V}$. The first state shown in figure 5, being a bifurcation point, belongs to the diffuse mode and is characterized by a nearly constant temperature of the front surface of the cathode. The second state, being still close to the bifurcation point, is characterized by smooth $3 \mathrm{D}$ perturbations. As the distance from the bifurcation point grows (the third state), the perturbations evolve into a well-defined spot at the edge of the front surface of the cathode. As the distance from the bifurcation point grows further and the current-voltage characteristic tends towards the region of small currents and high voltages (the fourth state), the spot shrinks and becomes brighter.

Evolution of the temperature distribution along the second, third and fourth 3D spot modes branching off from the diffuse mode (figures 6-8) is similar, the difference being that these modes are characterized by two, three and four spots (rather than one) positioned at the edge of the front surface of the cathode. The first state shown in figure 9, being a bifurcation point, belongs to the first axially symmetric spot mode and is characterized by a spot at the centre of the front surface of the cathode. As the distance from the bifurcation point grows, two additional spots emerge positioned opposite each other at the edge of the front surface.

Since the spots shrink when the current-voltage characteristics tend towards the region of small currents and high voltages, one should expect that in this region the interaction of each spot with distant parts of the cathode and with other spots (if they exist) becomes less important and the spot starts to behave like a solitary one. Let us check whether this can be observed in the modelling results.

According to general theory [5], the temperature inside a solitary spot is close to the limiting temperature $T_{2}$, which is the value of the surface temperature starting from which thermionic cooling exceeds the combined heating by the ions and plasma electrons and function $q$ turns negative. In this connection, the maximum temperature of the cathode surface and the corresponding limiting temperature $T_{2}$ for the first two $3 \mathrm{D}$ spot modes are shown in figure 10 . Note that the maximum temperature occurs at the edge of the front surface at $\phi=0$; if the distance from the bifurcation point is not too small and $\operatorname{spot}(\mathrm{s})$ are already well defined, this temperature may be interpreted as the maximum temperature inside the edge spot(s). Since $T_{2}=T_{2}(U)$, values of $T_{2}$ shown in figure 10 for each mode were calculated with the use of the currentvoltage characteristic, $U=U(I)$, of the respective mode: $T_{2}=T_{2}[U(I)]$. Also shown in figure 10 are the maximum temperature for the diffuse mode and the bifurcation points in which the 3D modes branch off from the diffuse mode.

The maximum temperature attained by the cathode in each mode remains below the upper limit of the cathode temperature as it should. The difference between these two temperatures is small, an indication that the spots indeed start behaving like solitary ones as the current decreases. 


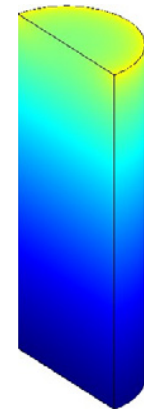

a

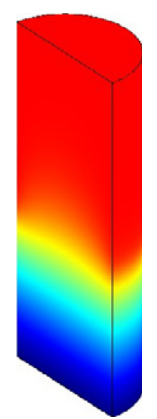

C

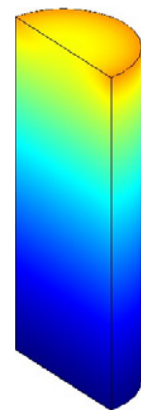

b

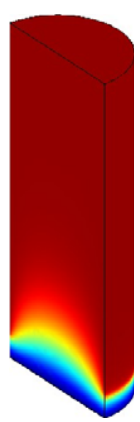

d

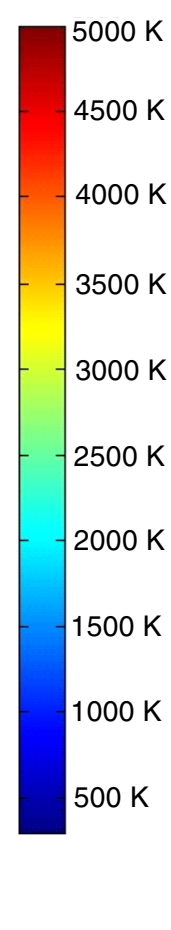

Figure 3. Temperature distribution for various states of the diffuse mode. $R=2 \mathrm{~mm}, h=10 \mathrm{~mm}$. (a) $I=15 \mathrm{~A}$. (b) $110 \mathrm{~A}$. (c) $10 \mathrm{kA}$. (d) $67 \mathrm{kA}$.

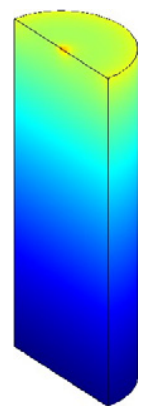

a

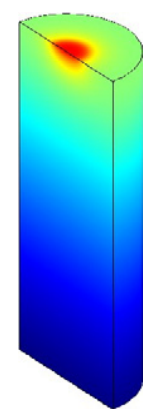

C

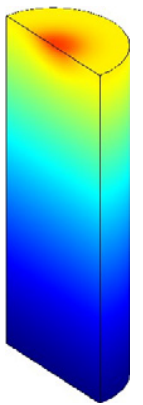

b

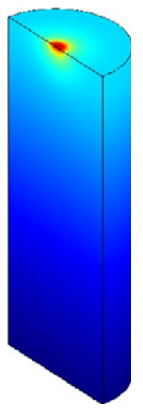

d
Figure 4. Temperature distribution for various states of the first axially symmetric spot mode. $R=2 \mathrm{~mm}, h=10 \mathrm{~mm}$. (a) $I=15 \mathrm{~A}$, low-voltage branch. (b) $110 \mathrm{~A}$, low-voltage branch. (c) $110 \mathrm{~A}$, high-voltage branch. (d) $41 \mathrm{~A}$, high-voltage branch. The temperature bar is shown in figure 3 .

Temperature and electric current density distributions along the line $\phi=0$ on the front surface of the cathode are shown in figures 11 and 12 , respectively, for the first two 3D spot modes at $U=40 \mathrm{~V}$. (In these figures, $r$ is the distance

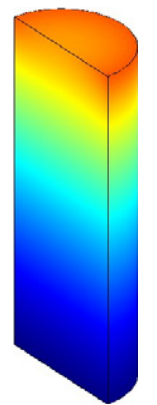

a

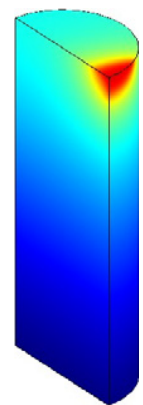

C

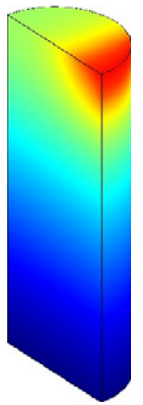

b

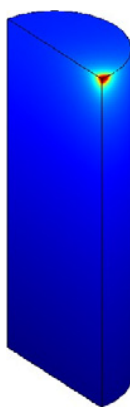

d
Figure 5. Temperature distribution for various states of the first 3D spot mode which branches off from the diffuse mode. $R=2 \mathrm{~mm}$, $h=10 \mathrm{~mm}$. (a) bifurcation point. (b) $U=12 \mathrm{~V}$. (c) $15 \mathrm{~V}$. (d) $40 \mathrm{~V}$. The temperature bar is shown in figure 3 .

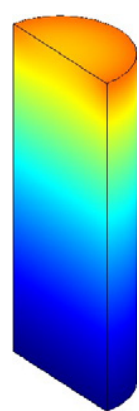

a

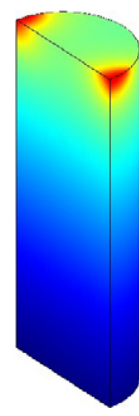

C

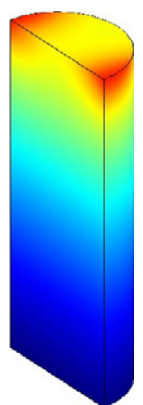

b

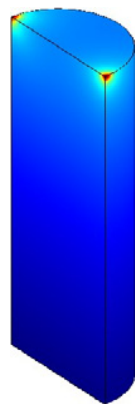

d
Figure 6. Temperature distribution for various states of the second 3D spot mode which branches off from the diffuse mode. $R=2 \mathrm{~mm}, h=10 \mathrm{~mm}$. (a) bifurcation point. (b) $U=12 \mathrm{~V}$. (c) $15 \mathrm{~V} .(d) 40 \mathrm{~V}$. The temperature bar is shown in figure 3.

from the centre of the cathode, so $r=2 \mathrm{~mm}$ corresponds to the edge. $U=40 \mathrm{~V}$ has been chosen as the highest value of the near-cathode voltage drop shown in figure 1 , i.e. $U=40 \mathrm{~V}$ corresponds to the lowest value of electric current for each 


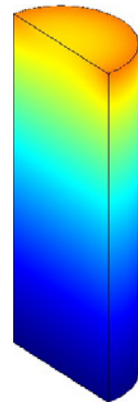

a

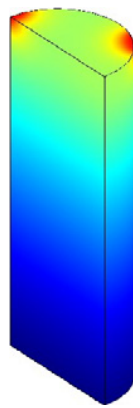

C

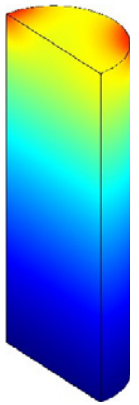

b

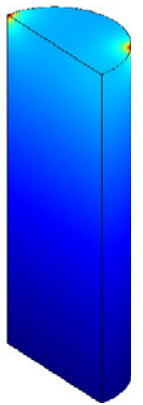

d

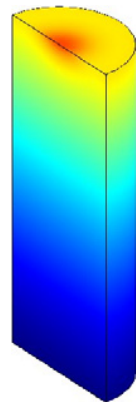

a

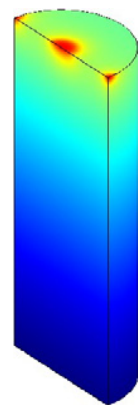

C

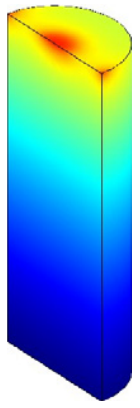

b

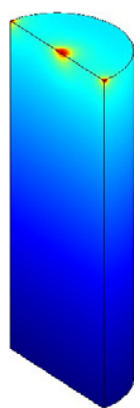

d
Figure 7. Temperature distribution for various states of the third 3D spot mode which branches off from the diffuse mode. $R=2 \mathrm{~mm}$, $h=10 \mathrm{~mm}$. (a) bifurcation point. (b) $U=12.7 \mathrm{~V}$. (c) $15 \mathrm{~V}$. (d) $40 \mathrm{~V}$. The temperature bar is shown in figure 3 .

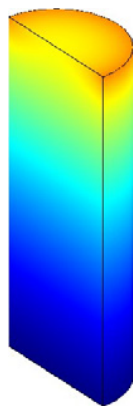

a

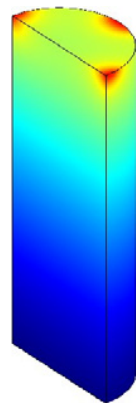

C

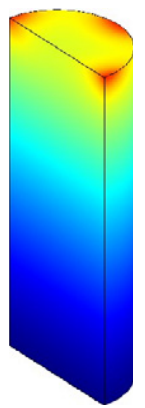

b

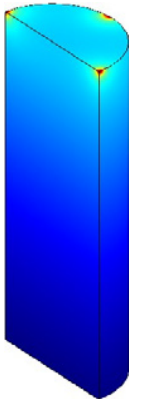

d
Figure 8. Temperature distribution for various states of the fourth $3 \mathrm{D}$ spot mode which branches off from the diffuse mode. $R=2 \mathrm{~mm}, h=10 \mathrm{~mm}$. (a) bifurcation point. (b) $U=13.4 \mathrm{~V}$.

(c) $15 \mathrm{~V}$. (d) $40 \mathrm{~V}$. The temperature bar is shown in figure 3 .

mode.) While within the spots the temperature distributions are close among themselves, they differ appreciably at large distances from the spot. The difference in the distributions of the electric current density within the spots is more pronounced
Figure 9. Temperature distribution for various states of the first 3D spot mode which branches off from the first axially symmetric spot mode. $R=2 \mathrm{~mm}, h=10 \mathrm{~mm}$. (a) bifurcation point.

(b) $U=15.2 \mathrm{~V}$. (c) $20 \mathrm{~V}$. (d) $40 \mathrm{~V}$. The temperature bar is shown in figure 3 .

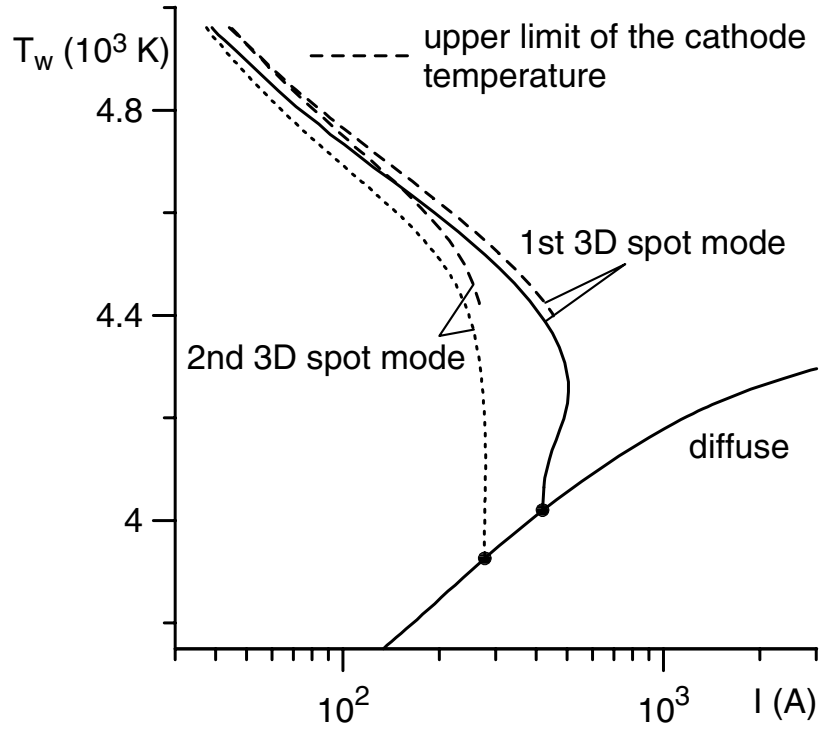

Figure 10. Maximum temperature of the cathode surface in different modes. $R=2 \mathrm{~mm}, h=10 \mathrm{~mm}$. Circles: bifurcation points.

than the difference in temperatures, which is a consequence of a strong dependence of function $j$ on $T_{w}$. One can conclude that the spots still continue to be affected by the cathode geometry under the conditions considered, i.e. they are not fully developed solitary spots yet.

In all the modes depicted in figures 4-9 spots are positioned either at the centre of the front surface, i.e. on the axis 


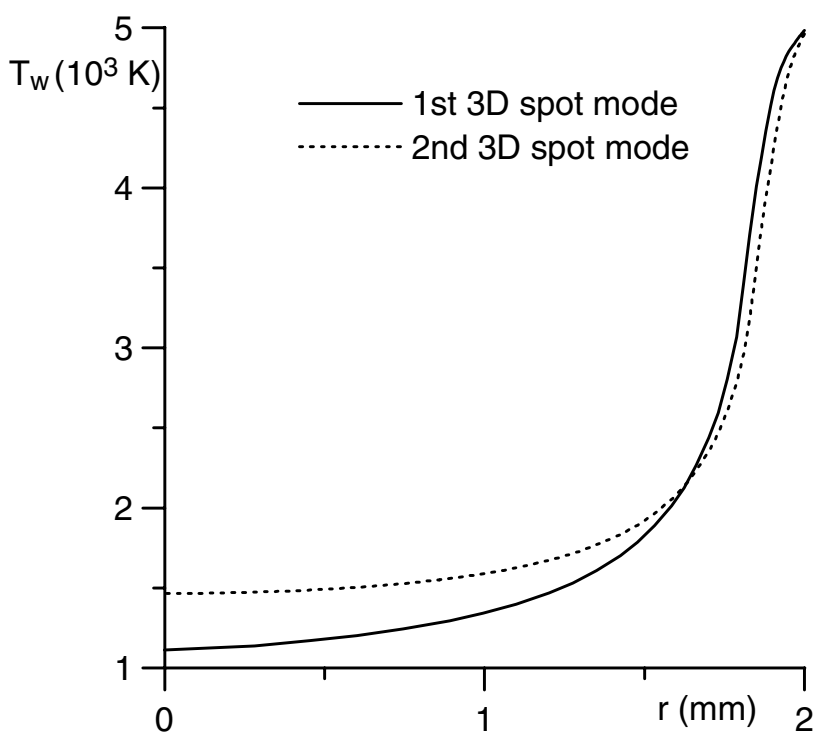

Figure 11. Distribution of the temperature within the spot. $R=2 \mathrm{~mm}, h=10 \mathrm{~mm} . U=40 \mathrm{~V}$.

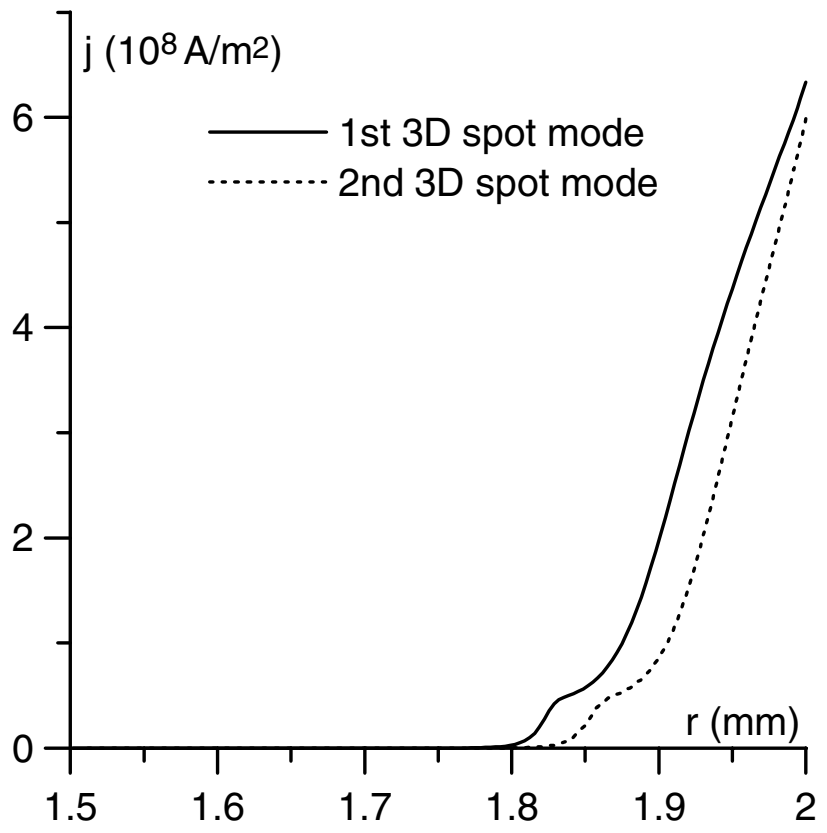

Figure 12. Distribution of the current density within the spot. $R=2 \mathrm{~mm}, h=10 \mathrm{~mm} . U=40 \mathrm{~V}$.

of symmetry of the cathode, or at the edge of the front surface. The fact that the edge of the front surface of a rod cathode, being a point where conditions for thermal-conduction heat removal are the worst, is one of the preferred points of spot attachment is well known from the experiment; see, e.g. [7, p 61], [15, p 1649]. On the other hand, one can expect that steady-state spots can also attach somewhere between the edge and the centre; however, corresponding modes have been left beyond the scope of the present paper. For example, the 3D mode branching off from the first axially symmetric spot mode at the bifurcation point associated with $k=1$ is likely to be a mode with two opposite spots, one of them being positioned at the edge and the other between the edge and the centre [6].

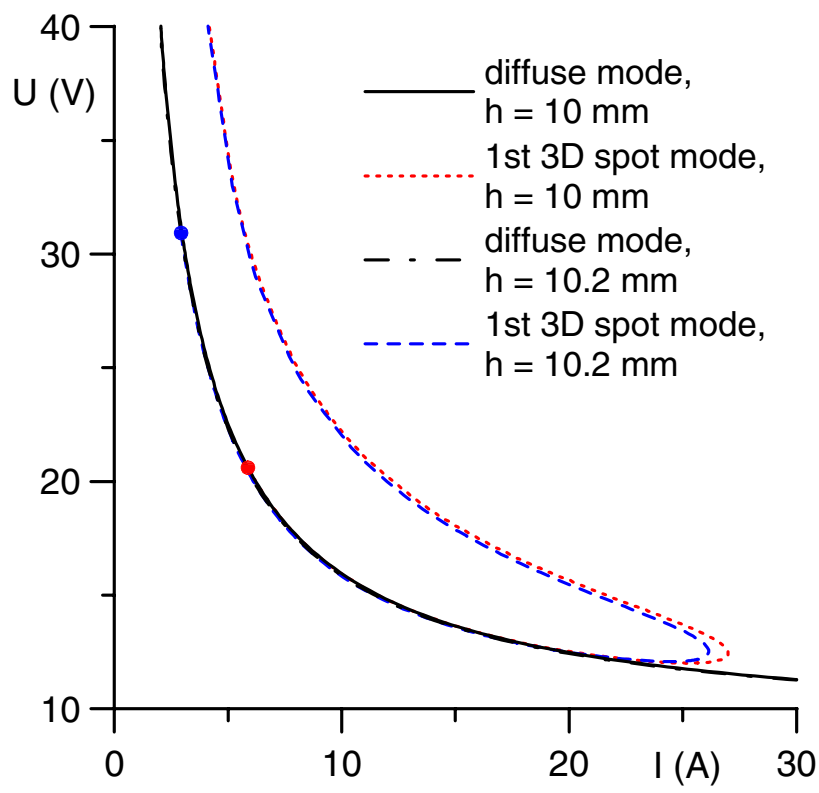

Figure 13. Current-voltage characteristics of the diffuse mode and the first 3D spot mode. $R=0.75 \mathrm{~mm}$. Circles: bifurcation points at which the first 3D mode branches off from the diffuse mode.

\section{Solutions for thin cathodes}

\subsection{Calculation results}

Cathodes best studied in the experiment (e.g. [3]) were appreciably slimmer and higher than those considered in the previous section and were operated at argon pressure higher than 1 bar. As a representative example, let us consider a tungsten cathode of radius $R=0.75 \mathrm{~mm}$ and height $h=20 \mathrm{~mm}$ operating in the argon plasma under the pressure of 2.6 bar.

Current-voltage characteristics of the diffuse mode and the first 3D spot mode on cathodes of radius $R=0.75 \mathrm{~mm}$ and heights $10 \mathrm{~mm}, 10.2 \mathrm{~mm}$ and $20 \mathrm{~mm}$, operating in the argon plasma under the pressure of $2.6 \mathrm{bar}$, are shown in figures 13 and 14. One can see from figure 13 that an increase in the cathode height from 10 to $10.2 \mathrm{~mm}$ produces no noticeable effect on the current-voltage characteristic of the diffuse mode: the corresponding curves coincide. The effect produced on the characteristic of the first 3D spot mode is more considerable. First, the turning point is slightly shifted in the direction of small currents. Second, there is a considerable shift in the position of the bifurcation point at which the first 3D spot mode branches off from the diffuse mode: it changes from 5.9 to $2.9 \mathrm{~A}$. The current-voltage characteristics of the cathode of height $h=20 \mathrm{~mm}$, shown in figure 14, are qualitatively similar to those shown in figure 13; the difference is that the first bifurcation point has moved into the region of very high voltages (well in excess of $100 \mathrm{~V}$ ) and is absent from the graph, while the turning point has moved further into the region of lower currents.

One can view the first 3D spot mode as consisting of two branches, a high-voltage branch which exists in the current range $0<I \leqslant I_{\mathrm{t}}$ (i.e. from zero up to a current $I_{\mathrm{t}}$ corresponding to the turning point) and a low-voltage branch which exists in the current range $I_{\mathrm{b}} \leqslant I \leqslant I_{\mathrm{t}}$ (i.e. between the first bifurcation point and the turning point). In the case of a wide cathode, shown in figures 1 and 2, the first bifurcation 
3D modelling of heating of thermionic cathodes

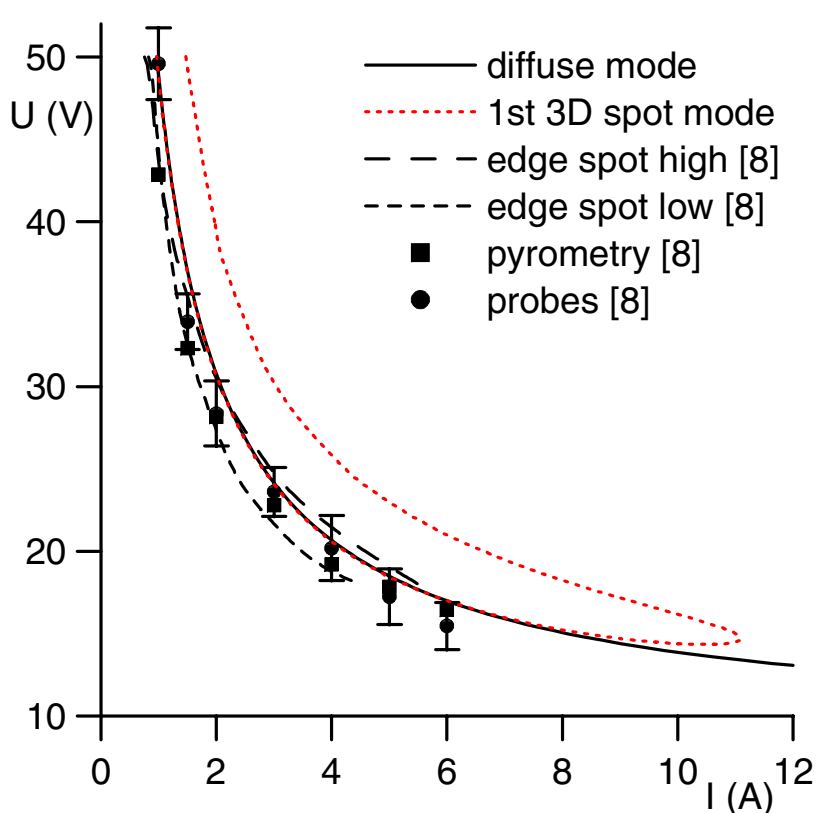

Figure 14. Current-voltage characteristics of the diffuse mode and of the first 3D spot mode. $R=0.75 \mathrm{~mm}, h=20 \mathrm{~mm}$. Points: experimental data on near-cathode voltage obtained by means of pyrometric and probe measurement.

point and the turning point are not very distant and the lowvoltage branch is not well pronounced (i.e. the current range $I_{\mathrm{b}} \leqslant I \leqslant I_{\mathrm{t}}$ in which this branch exists is rather narrow). As the cathode becomes thinner, the current range between the first bifurcation point and the turning point of the first 3D spot mode expands and the low-voltage branch becomes well pronounced. The greater part of the current-voltage characteristic of the low-voltage branch on a thin cathode is virtually indistinguishable from the characteristic of the diffuse mode.

The current-voltage characteristic of the first 3D spot mode on a thin cathode shown in figure 14 is very similar to the characteristic of the first axially symmetric spot mode on a wide cathode calculated and discussed in [5] and shown also in figures 1 and 2 of the present work. An analysis of the temperature distributions on the first 3D spot mode on a thin cathode also reveals such similarity: the spot is hot and well pronounced on the high-voltage branch and is colder and somewhat diffuse on the low-voltage branch, similarly to what was found in calculations of the first axially symmetric spot mode on a wide cathode [5] and is shown in figure 4 of the present work.

In [8], simulation results have been reported for conditions similar to those of figure 14 . Three modes have been found: a diffuse mode and two edge spot modes termed a lowtemperature spot mode and a high-temperature spot mode. Current-voltage characteristics of the spot modes taken from figure 7 of [8] are depicted in figure 14. Both modes cease to exist (break off) as the arc current exceeds certain values, which are about 4.5 and 5.5 A for low and high-temperature spot modes, respectively.

The pattern of spot mode solutions found in [8] does not conform to the pattern established in [1,6] and is not typical for multiple solutions (normally a solution does not just disappear but rather turns back or joins another solution). Results of the present work do not confirm this pattern: figure 14 suggests

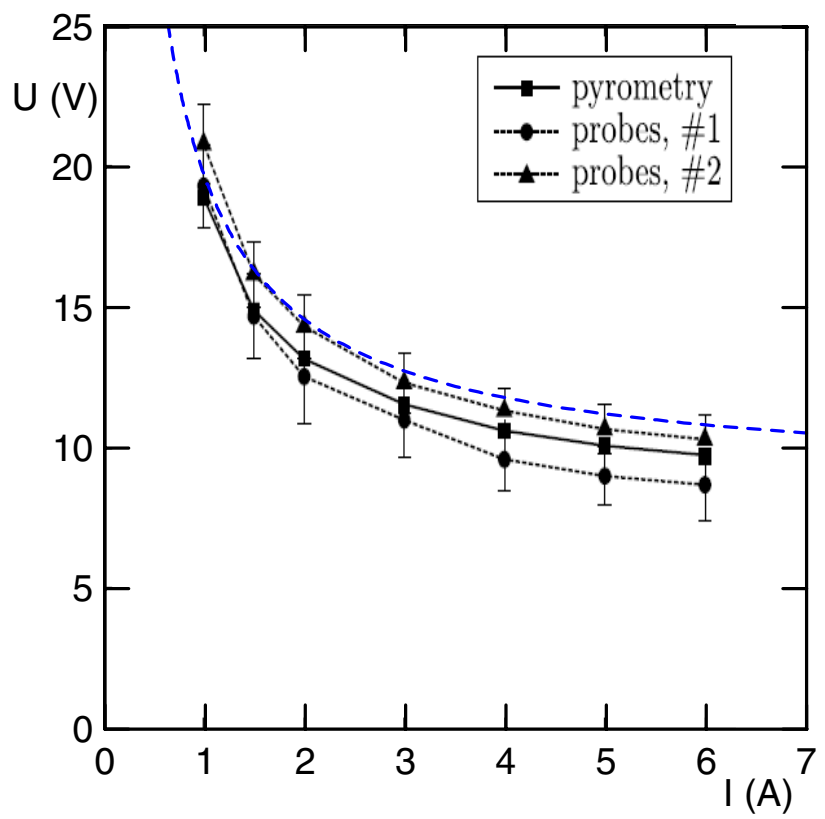

Figure 15. Current-voltage characteristics of the diffuse mode. $R=0.3 \mathrm{~mm}, h=20 \mathrm{~mm}$. Points: experimental data on near-cathode voltage obtained by means of pyrometric and probe measurement [8]. Dashed line: modelling of the present work.

that spot mode solutions have been found in [8] in only a part of their existence regions; these solutions do not represent separate modes but rather are parts of the high-voltage and low-voltage branches of the first 3D spot mode; the break off of spot mode solutions reported in [8] is a numerical and not a physical effect.

In addition to the above-discussed difference in pattern, the spot mode solutions of the present work and of [8] also manifest quantitative differences. In particular, one can see from figure 14 that values of the near-cathode voltage drop corresponding to each branch of the first 3D spot mode, calculated in the present work, are somewhat higher than those reported in [8]. This difference may originate in low accuracy of numerical simulation and/or differences in functions $q\left(T_{w}, U\right)$ and $j\left(T_{w}, U\right)$ used in the present calculations and in [8]. In order to illustrate the role of differences in functions $q\left(T_{w}, U\right)$ and $j\left(T_{w}, U\right)$, we reproduce in figure 15 data on the diffuse mode shown in figure 12 of [8]. The experimental points are connected by the solid or dotted lines and represent data on the cathode fall derived from pyrometric temperature measurements or data obtained by different probe measurements performed immediately before and after the temperature measurements. The current-voltage characteristic calculated by the authors [8] coincides exactly with the characteristic given by pyrometric measurements; modelling results of the present work are depicted by the dashed line. One can see that the near-cathode voltage drop calculated in the present work again is somewhat higher than that calculated by the authors [8]. Since the (axially symmetric) numerical simulation of the diffuse mode is quite accurate, one should conclude that this difference stems from differences in functions $q\left(T_{w}, U\right)$ and $j\left(T_{w}, U\right)$ used in the calculations. It should be emphasized, however, that both the current-voltage characteristic of the present work and that calculated by the authors [8] conform to the experimental 
data within experimental error, which adds credibility to the approach considered.

\subsection{Comparison with the experiment}

A quantitative agreement of the present model with the experiment for the case of diffuse mode has already been established [2,3]; figure 15 in this sense represents just one more example. As far as the spot mode is concerned, quantitative agreement can hardly be expected. On one hand, this is a consequence of limitations of the model of nonlinear surface heating, in which only the voltage drop in a thin near-cathode layer is considered. The latter is likely to be a reasonable approximation for the diffuse mode; however, in the case of a spot mode the electron temperature in the spot is quite high and perturbations are not necessarily confined to a thin near-cathode layer. There are also difficulties on the experimental side: even such a basic quantity as the nearcathode voltage drop cannot be measured directly; a cathode spot is a difficult object for experimental investigation due to its small dimensions and ability to appear at an arbitrary position; additional difficulties are caused by the poor reproducibility of the diffuse-spot transition.

The absence of quantitative agreement between the model of nonlinear surface heating and the experiment in the case of the spot mode can be immediately seen from the fact that the near-cathode voltage drop measured in the spot mode is lower than that for the diffuse mode and the differences are about $6 \mathrm{~V}$ at $1.5 \mathrm{~A}$ and $1 \mathrm{~V}$ at $6 \mathrm{~A}$ [15]. No such differences have been detected either in the present modelling or in [8]: the voltage drop on the low- or high-voltage branches of the spot mode is close to or, higher respectively, than that in the diffuse mode. On the other hand, the difference between modelling and the experiment is not dramatic, which can be seen from figure 14 where experimental data on the near-cathode voltage drop in the spot mode taken from figure 17 of [8] are depicted. One can see that the current-voltage characteristic of the low-voltage branch of the first 3D spot mode is in reasonable agreement with the experiment, while values of the near-cathode voltage drop corresponding to the high-voltage branch exceed the experimental values.

It is interesting to note that the latter does not apply to results of the modelling [8]: one can see from figure 14 that the current-voltage characteristics of both the low- and highvoltage branches calculated in [8] are in reasonable agreement with the experiment. However, the authors [8] believe that it is the low-voltage branch of the spot mode which is observed in the experiment since the cathode surface temperature which they calculated for the high-voltage branch is far beyond the melting temperature of tungsten.

\section{Stability considerations}

\subsection{General reasoning}

It has been shown in the preceding sections that the problem of current transfer to thermionic cathodes has a whole 'zoo' of very diverse steady-state solutions. A question arises as to which of these solutions are stable and can be observed in the experiment. Unfortunately, a theory of stability of solutions to the problem considered is absent.

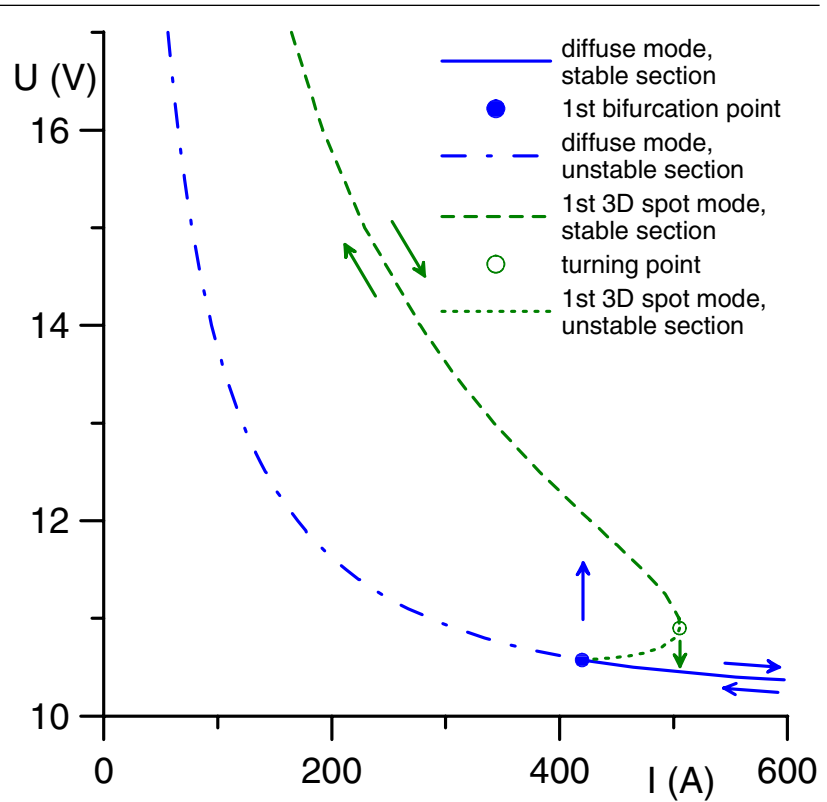

Figure 16. Transition between the diffuse mode and the first $3 \mathrm{D}$ spot mode. $R=2 \mathrm{~mm}, h=10 \mathrm{~mm}$.

The authors [8] assumed, on the basis of arguments stemming from Steenbeck's principle of minimum voltage (power) for discharges with a fixed current (e.g. [16, pp 1845]), that a mode with the lowest near-cathode voltage drop is the preferred one. However, Steenbeck's minimum principle bears no relation to fundamental physical principles; although some authors (e.g. [17-19]) seem to believe that it can be proved by methods of thermodynamics, we have not encountered any such proof in the literature. Equally, Steenbeck's minimum principle bears no relation to stability theory. In summary, this principle is just an arbitrary assumption and arguments based on this principle are not convincing.

In [1], hypotheses concerning stability of different steadystate solutions have been put forward on the basis of general trends typical for nonlinear dissipative systems. These hypotheses can be summarized as follows. The diffuse mode is stable against small perturbations beyond the first bifurcation point (at $I>I_{\mathrm{b}}, I_{\mathrm{b}}$ being the current corresponding to the first bifurcation point) and unstable at lower currents (at $I<I_{\mathrm{b}}$ ). The bifurcation which occurs in the first bifurcation point is subcritical under the conditions considered, i.e. the first 3D spot mode branches from the diffuse mode into the range $I>I_{\mathrm{b}}$ in which the diffuse mode is stable. By analogy with other problems in which subcritical bifurcations occur (e.g. [20]), one could expect that the initial section of the first 3D spot mode is unstable. One could also expect that the change in stability of the first 3D spot mode occurs at the turning point: this mode is stable beyond the turning point and unstable at lower voltages. The second and all the subsequent $3 \mathrm{D}$ modes are expected to be unstable.

In the framework of these hypotheses, the transition between the diffuse and spot modes under conditions of figure 1 occurs as shown in figure 16. At small currents, the discharge burns in the spot mode. With an increase of current, a transition to the diffuse mode occurs at $I=I_{\mathrm{t}}$ (in the turning point). At high currents, the discharge can burn only in the diffuse mode. With a decrease in current, a transition to the first 3D spot mode 
3D modelling of heating of thermionic cathodes

Table 1. Ranges of existence and/or stability of the diffuse mode and the high-voltage branch of the first 3D spot mode. $R=0.75 \mathrm{~mm}$.

\begin{tabular}{llll}
\hline $\begin{array}{l}\text { Cathode height } \\
(\mathrm{mm})\end{array}$ & $\begin{array}{l}\text { Stable high-voltage branch } \\
\text { of the first 3D spot mode, } \\
\text { unstable diffuse mode(A) }\end{array}$ & $\begin{array}{l}\text { Stable high-voltage branch } \\
\text { of the first 3D spot mode, } \\
\text { stable diffuse mode(A) }\end{array}$ & $\begin{array}{l}\text { Stable diffuse } \\
\text { mode(A) }\end{array}$ \\
\hline 10 & $I<5.9$ & $5.9<I<27.0$ & $I>27.0$ \\
10.2 & $I<2.9$ & $2.9<I<26.1$ & $I>26.1$ \\
20 & - & $I<11.1$ & $I>11.1$ \\
\hline
\end{tabular}

occurs at $I=I_{\mathrm{b}}$. Since $I_{\mathrm{b}}<I_{\mathrm{t}}$, the transition between the diffuse mode and the first $3 \mathrm{D}$ spot mode manifests hysteresis in the current range $I_{\mathrm{b}} \leqslant I \leqslant I_{\mathrm{t}}$.

\subsection{Comparison with the experiment}

When applied to thin cathodes, the above hypothesizing suggests that the low-voltage branch of the first 3D spot mode is unstable and the high-voltage branch is stable.

For convenience, ranges of existence and/or stability of the diffuse mode and the high-voltage branch of the first 3D spot mode under conditions of figures 13 and 14 (a tungsten cathode of radius $R=0.75 \mathrm{~mm}$ operating in the argon plasma under pressure of 2.6 bar) are summarized in table 1. For the cathode of height $h=20 \mathrm{~mm}$, which is a representative experimental example, the diffuse mode is stable. (More precisely, the diffuse mode is stable in the whole current range in which it is shown in figure 14; it loses stability at very low currents corresponding to very high voltages well in excess of $100 \mathrm{~V}$.) Thus, there are two modes in the range $I \leqslant I_{\mathrm{t}}=11.1$ A which are stable against small perturbations, the diffuse mode and the high-voltage branch of the first spot mode. Only the diffuse mode is possible at $I>11.1 \mathrm{~A}$.

Experiments on diffuse-spot transition are usually performed in a limited current and voltage range, say $I \leqslant 10 \mathrm{~A}$ and $U \leqslant 100 \mathrm{~V}$. On the basis of the above hypothesizing, one should assume that two stable (against small perturbations) modes exist in the whole range of conditions of such experiments, the diffuse mode and the high-voltage branch of the spot mode. If the experiment is performed under well-controlled quasi-stationary conditions, a mode which has occurred immediately after the ignition of the discharge will be maintained during the whole experimental run. The mode change can occur only due to finite perturbations. For example, if experimental parameters (e.g. the arc current or the plasma pressure) have changed rapidly, it is possible that the discharge will occur, after the steady-state has been recovered, not in the original mode but in the other one. If the mode change is systematically observed in such an experiment under quasistationary conditions, it means that the experiment is not wellcontrolled. For example, non-uniformities of the cathode surface (e.g. protrusions) can provoke mode changes which are not described by the above reasoning.

The above hypothesizing explains the general trend that the transition between the diffuse and spot modes is difficult to reproduce in the experiment. On the other hand, thishypothesizing predicts that the low-voltage branch of the first 3D spot mode is unstable and the high-voltage branch is stable, which contradicts the indication that it is the low-voltage branch of the first 3D spot mode that seems to occur in the experiment (see discussion in section 4.2). This contradiction casts doubts on the part of the above hypothesis concerning (in)stability of the two branches of the first 3D spot mode and suggests that in reality the low-voltage branch is stable while the high-voltage branch is unstable. It should be emphasized that such a suggestion, while resolving the contradiction, does not interfere with the above conclusion in the absence of a reproducible transition between diffuse and spot modes under typical experimental conditions if the experiment is wellcontrolled.

One can conclude that the available theoretical information on stability of different modes is clearly insufficient and an accurate analysis of stability of steady-state solutions is required. Another step which could prove highly useful for understanding the general pattern and stability of different modes would be an experimental investigation of diffuse and spot modes under conditions when the interval $I_{\mathrm{b}} \leqslant I \leqslant I_{\mathrm{t}}$ is inside the current range being investigated.

\section{Conclusions}

Numerical investigation of steady-state current transfer from a high-pressure arc plasma to a cylindrical thermionic cathode has revealed a whole 'zoo' of very diverse modes. Detailed results are given for the first four 3D modes branching off from the diffuse mode and for the first 3D mode branching off from the first axially symmetric spot mode. Each 3D mode is calculated in the whole domain of its existence, from the bifurcation point in which it branches off from an axially symmetric mode down to very low currents. One can clearly observe the evolution of a temperature distribution along the cathode surface from a rather smooth one in the vicinity of the bifurcation point to one with well-defined spots at large distances from the bifurcation point. At very low currents, the corresponding near-cathode voltage drop infinitely increases and each spot shrinks and its behaviour approaches that of a solitary spot. The physics of 3D spots is similar to the physics of axially symmetric spots.

The numerical results obtained confirm the general pattern of current-voltage characteristics of various modes suggested in $[1,6]$ on the basis of bifurcation analysis and general considerations. It is shown, in particular, that a 3D spot mode cannot just terminate but rather turns back or joins an axially symmetric mode. It is also shown that the current range in which 3D spot modes exist is limited from above: $I \leqslant I_{\mathrm{t}}$, where $I_{\mathrm{t}}$ is the current value corresponding to the turning point of the first 3D spot mode. Values of the near-cathode voltage drop calculated for the low-voltage branch of the first 3D spot mode conform to experimental data for the spot mode available in the literature.

Hypotheses on stability of steady-state solutions based on general trends typical for nonlinear dissipative systems provide 
an explanation of the fact that the transition between diffuse and spot modes is difficult to reproduce in the experiment: if a thin cathode is operated in a limited current range, it is likely that both the diffuse mode and one of the branches of the first 3D spot mode will be stable against small perturbations in the whole range of experimental conditions. On the other hand, these hypotheses do not explain the indication that it is the lowvoltage branch of the first 3D spot mode that seems to occur in the experiment. Thus, the question of stability of steadystate solutions remains open: an accurate stability analysis is required. Another step which could prove highly useful would be an experimental investigation of diffuse and spot modes under conditions where both the first bifurcation point and the turning point of the first 3D spot mode are inside the current range being investigated.

\section{Acknowledgments}

The work was performed within activities of the project POCI/FIS/60526/2004 'Modes of current transfer to cathodes of high-pressure arc discharges and their stability' of FCT, POCI 2010 and FEDER and of the action 529 of the programme COST of the EC. MC acknowledges financial support granted by the action COST-529 towards his stay at Universidade da Madeira.

\section{References}

[1] Benilov M S 1998 Phys. Rev. E 58 6480-94

[2] Benilov M S and Cunha M D 2002 J. Phys. D: Appl. Phys. 35 $1736-50$
[3] Nandelstädt D, Redwitz M, Dabringhausen L, Luhmann J, Lichtenberg S and Mentel J 2002 J. Phys. D: Appl. Phys. 35 1639-47

[4] Bötticher R and Bötticher W 2000 J. Phys. D: Appl. Phys. 33 367-74

[5] Benilov M S and Cunha M D 2003 J. Phys. D: Appl. Phys. 36 603-14

[6] Benilov M S and Cunha M D 2003 Phys. Rev. E 68056407

[7] Bötticher R, Graser W and Kloss A 2004 J. Phys. D: Appl. Phys. 37 55-63

[8] Dabringhausen L, Langenscheidt O, Lichtenberg S, Redwitz M and Mentel J 2005 J. Phys. D: Appl. Phys. 38 $3128-42$

[9] Benilov M S, Cunha M D and Naidis G V 2005 Plasma Sources Sci. Technol. 14 517-524

[10] http://www.arc_cathode.uma.pt

[11] Benilov M S and Marotta A 1995 J. Phys. D: Appl. Phys. 28 1869-82

[12] White G K and Minges M L 1997 Int. J. Thermophys. 18 1269-327

[13] Touloukian Y S, Powell R W, Ho C Y and Clemens P G 1970 Thermal Conductivity. Metallic Elements and Alloys (Thermophysical Properties of Matter vol 1) (New York-Washington: IFI/Plenum)

[14] Yih S W H and Wang C T 1979 Tungsten: Sources, Metallurgy, Properties, and Applications (New York: Plenum)

[15] Lichtenberg S, Nandelstädt D, Dabringhausen L, Redwitz M, Luhmann J and Mentel J 2002 J. Phys. D: Appl. Phys. 35 $1648-56$

[16] Raizer Yu P 1991 Gas Discharge Physics (Berlin: Springer)

[17] Haidar J 1999 J. Phys. D: Appl. Phys. 32 263-72

[18] Schmitz H and Riemann K-U 2002 J. Phys. D: Appl. Phys. 35 1727-35

[19] Hantzsche E 2003 IEEE Trans. Plasma Sci. 31 799-808

[20] Benilov M S 1992 Phys. Lett. A 169 57-61 